\title{
Search for new T Tauri stars in the Cepheus-Cassiopeia region
}

\author{
K. Tachihara ${ }^{1}$, R. Neuhäuser ${ }^{1}$, M. Kun ${ }^{2}$, and Y. Fukui ${ }^{3}$ \\ 1 Astrophysikalisches Institut und Universitäts-Sternwarte Jena, Schillergäßchen 2-3, 07745, Jena, Germany \\ e-mail: tatihara@astro.uni-jena.de \\ 2 Konkoly Observatory of Hungarian Academy of Sciences, Budapest, Hungary \\ 3 Department of Astrophysics, Nagoya University, Chikusa-ku, Nagoya, 464-8602, Japan
}

Received 18 October 2004 / Accepted 18 February 2005

\begin{abstract}
The Cepheus-Cassiopeia star-forming region has been searched for new T Tauri stars (TTSs) based on the ROSAT all sky survey (RASS). Optical spectroscopic observations were carried out toward 45 GSC stellar counterparts of RASS sources looking for the Li absorption line (6708 $\AA$ ), a sign of youth. The detection of this line resulted in finding 11 to 16 new TTSs in this region. Using follow-up optical photometric observations and evolutionary models these Li-rich stars are revealed to be young low-mass stars. The most of the young stars are separated from the ${ }^{13} \mathrm{CO}$ molecular clouds by $\sim 10 \mathrm{pc}$, significantly more than in Chamaeleon. A group of the new TTSs are isolated from the $\mathrm{CO}$ clouds and distributed inside the previously determined $\mathrm{CO}$ void. Possible formation scenarios including the interaction with the supernova shock for the isolated TTSs are discussed.
\end{abstract}

Key words. ISM: clouds - ISM: molecules - stars: formation - stars: late-type - stars: pre-main sequence - X-rays: stars

\section{Introduction: The Cepheus-Cassiopeia star-forming region}

Large-scale X-ray surveys are a powerful method to search for T Tauri stars (TTSs) that are widely spread over starforming regions (SFRs). Spectroscopic follow-up observations towards the X-ray sources found by the ROSAT X-ray satellite have been carried out in nearby SFRs such as Taurus (Neuhäuser et al. 1997), Chamaeleon (Alcalá et al. 1995), and Lupus (Wichmann et al. 1997), most of which are thought to belong to the Gould belt. These surveys found a large number of T Tauri stars (see Neuhäuser 1997 for a review). One of the interesting results of the X-ray selected TTS surveys is that a large number of TTSs have been found far away from the molecular clouds. Because of their youth ( $\$ \mathrm{a}$ few $\times 10^{7} \mathrm{yr}$ ), they are supposed to be distributed within $\sim 10 \mathrm{pc}$ of their birthplaces. Nonetheless, many so-called "isolated TTSs", separated from the molecular clouds by more than a few tens of pc, are found, for example, south of the Taurus (Neuhäuser et al. 1997) and $\eta$ Cha clusters (Mamajek et al. 1999), even though many of those south of Taurus were later reported to be active binaries (Torres et al. 2002). Recent studies suggest that such isolation is likely caused by in situ star formation in small clouds and their subsequent rapid dissipation (e.g., Mizuno et al. 1998; Tachihara et al. 2001).

Cepheus-Cassiopeia (Cep-Cas) is a nearby SFR located at $l \sim 100^{\circ}-140^{\circ}$ and $b \sim 5^{\circ}-25^{\circ}$. The region has been surveyed extensively for molecular clouds in ${ }^{12} \mathrm{CO}$ by Grenier et al. (1989) who found a total molecular mass of $\sim 10^{5} M_{\odot}$. They reported that the cloud distribution shows a $\mathrm{CO}$ void which contains an excess of soft X-rays and radio continuum, suggesting a hot bubble created by a supernova. A distance estimation to several dark clouds have been carried out by measuring the colour excess or utilizing star counts (Kun \& Prusti 1993; Kun et al. 1994; Kun 1998; Obayashi et al. 1998). Yonekura et al. (1997) surveyed the region in ${ }^{13} \mathrm{CO}$ and detected 188 distinct molecular clouds. They obtained different radial velocities suggesting that layers at different distances are overlapping. Most of them at higher Galactic latitude $\left(b \gtrsim 8^{\circ}\right)$ are nearby, at a distance $160<d<440 \mathrm{pc}$. Star formation is active there and in the region called the Cepheus flare, where more than 100 young stellar objects (YSOs) were found (Kun 1998; Kun et al. 2000). A cometary-shape cloud, LDN 1251, is forming young stars with molecular outflows in the dense cores at the dense head part (Sato \& Fukui 1989; Sato et al. 1994), which suggests triggered star formation.

Even though the CO survey revealed the large scale molecular cloud distribution, the search for YSOs was limited to specific clouds. In order to make a comprehensive YSO census, we obtained spectroscopic observations looking for the lithium absorption lines $(\lambda=6708 \AA)$ towards the ROSAT X-ray point sources whose nature was unknown. Our observations are described in Sect. 2. In Sect. 3 we present the derived physical parameters of the newly found YSOs. We discuss the star formation history of this region in the light of our new results in Sect. 4.

\section{Observations}

\subsection{Target selection}

TTSs are known to be X-ray emitters whose plasma temperature is about $k T \sim 1 \mathrm{keV}$ (Feigelson \& DeCampli 1981 for 
Einstein data; Neuhäuser et al. 1995 for ROSAT data). For the ROSAT X-ray survey, they are characterized as point sources with hard X-ray spectra. In order to identify target candidates, we first extracted the point sources from the ROSAT all sky image with a maximum likelihood of existence $(M L)$ larger than $7.4(M L=7.4$ corresponds to a probability of existence of 0.999 and 3.5 Gaussian sigma significance, the best choice from a trade-off study in Neuhäuser et al. 1995), and the hardness ratios, defined as follows, are greater than 0 . If $Z_{\mathrm{s}}, Z_{\mathrm{h} 1}$, and $Z_{\mathrm{h} 2}$ are the count rates in the bands soft $(0.1$ to $0.4 \mathrm{keV})$, hard 1 ( 0.5 to $0.9 \mathrm{keV})$, and hard 2 (0.9 to $2.1 \mathrm{keV})$, respectively, then

$$
H R 1=\frac{Z_{\mathrm{h} 1}+Z_{\mathrm{h} 2}-Z_{\mathrm{s}}}{Z_{\mathrm{h} 1}+Z_{\mathrm{h} 2}+Z_{\mathrm{s}}} \quad \& \quad H R 2=\frac{Z_{\mathrm{h} 2}-Z_{\mathrm{h} 1}}{Z_{\mathrm{h} 2}+Z_{\mathrm{h} 1}}
$$

i.e., hardness ratios range from -1 to +1 , and hardness ratio errors can be larger than 2. As calculated with an assumption of the single temperature Raymond-Smith spectra by Neuhäuser et al. (1995), these criteria for the hardness ratios ensure that the plasma temperature is $k T \geq 0.8 \mathrm{keV}$ and the absorbing hydrogen column density is $20 \leq \log \left(N_{\mathrm{H}} / \mathrm{cm}^{2}\right) \leq 22$ for $0.8 \mathrm{keV} \leq k T \leq 1.4 \mathrm{keV}$.

These hard X-ray point sources were selected from a region of $19^{\mathrm{h}} 30^{\mathrm{m}}<\alpha_{2000}<3^{\mathrm{h}} 00^{\mathrm{m}},+64^{\circ}<\delta_{2000}<+82^{\circ}(1422$ objects). Next we searched the HST Guide Star Catalogue (GSC) for optical counterparts of the X-ray sources within 18" separation whose nature (whether they are stars or extragalactic objects, pre-main sequence stars or not, and their spectral types) was unknown (277 objects). Among them, for observational convenience, relatively bright (GSC magnitude $\leq 13$ mag) stars were used (185 objects). We preferentially chose the sources with smaller optical-to-X-ray separations (41 were selected out of 98 objects whose separations are $\leq 10^{\prime \prime}$, and 5 were selected out of the remaining 87 objects whose separations are $>10^{\prime \prime}$ ) to be observed with optical spectroscopy. The X-ray properties of the selected 46 objects are listed in Table 1. The X-ray flux, $f_{\mathrm{X}}$, is derived as

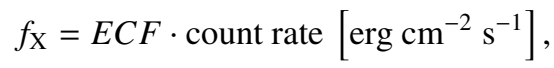

where $E C F$ is energy conversion factor given as

$$
E C F=(5.30 \cdot H R 1+8.31) \times 10^{-12}\left[\mathrm{erg} \mathrm{cm}^{-2} \mathrm{cts}^{-1}\right]
$$

according to Schmitt et al. (1995). The ROSAT All-Sky Survey Faint Source Catalog was published after our observing run (Voges et al. 2000), and all our targets are included therein or in the ROSAT All-Sky Bright Source Catalogue (Voges et al. 1999).

The properties of the target GSC stars (optical position, $V$ magnitude $\left(m_{V}\right)$, offset between $\mathrm{X}$-ray and optical positions and $\mathrm{X}$-ray to $V$-band magnitude flux ratio) are shown in Table 2. The optical flux, $f_{V}$, is calculated as

$f_{V}=10^{-0.4 \cdot(V+13.42)} \mathrm{erg} \mathrm{cm}^{-2} \mathrm{~s}^{-1}$

with $V$ in mag.

\subsection{Optical spectroscopic observation}

A spectroscopic analysis is the best method to establish the nature of the selected stars. In particular, Li I absorption is a good indicator of youth. Optical spectra of the selected candidate stars were taken with the Calar Alto $2.2 \mathrm{~m}$ telescope with the CAFOS spectrograph between 1 and 4 September 2000. At some target positions more than one object was found within a circle of 40", which corresponds to the RASS spatial resolution. We put the slit on the two objects in order to obtain spectra of both. We distinguish the components of these candidates by suffixes, $\mathrm{c} 1$ for the brighter ones and $\mathrm{c} 2$ for the fainter ones (c for candidate). The slit width was $\sim 1.5^{\prime \prime}$ which gives a 3-pixel wavelength resolution of $6.1 \AA$ in the range of $4500-8000 \AA$. For the wavelength calibration, spectra of a $\mathrm{Hg}$-He lamp were taken after every two or three target stars. The typical exposure time was $300 \mathrm{~s}$ for bright stars $\left(m_{V}=10 \mathrm{mag}\right)$ and $2200 \mathrm{~s}$ for faint stars $\left(m_{V}=13 \mathrm{mag}\right)$. Data reduction was carried out in IRAF.

For identification of the Li line, we compare its strength with that of the nearby $\mathrm{Ca}$ I $\lambda 6718$ line, which was visible in most of the spectra. If the Li I line was stronger than the Ca I line, we classified the star as Li-rich. Soderblom et al. (1993) measured the equivalent width of the $\mathrm{Li}$ I $(W(\mathrm{Li}))$ and the Ca I $(W(\mathrm{Ca} 6718))$ lines of the Pleiades stars. They showed that $W(\mathrm{Ca} 6718)$ is about the same as or larger than $W(\mathrm{Li})$ for various spectral types of Pleiades stars, while $W(\mathrm{Li})$ is more dispersed where $T_{\text {eff }}<5500 \mathrm{~K}$ (see also Fig. 3). Because the $\mathrm{Li}$ abundance, unlike $\mathrm{Ca}$, decreases monotonically with stellar age due to proton-proton capture, the comparison of the line strengths gives a reasonable initial assumption of whether our stars are younger than the Pleiades or not. A star with more Li than a Pleiades member of the same spectral type is younger than the Pleiades, i.e. pre-main sequence (PMS). The equivalent widths of the Li as well as Na I (5890 $)$ ), Ca I (6162 $\AA$ ) and $\mathrm{H} \alpha(6563 \AA)$ lines were measured by the IRAF SPLOT task.

\subsection{Optical photometric observation}

For all the candidate young stars that show Li absorption lines as strong as the $\mathrm{Ca}$ line, we performed optical photometric observations in the $V$ and $I_{\mathrm{C}}$ bands with the $1 \mathrm{~m}$ RCC telescope at the Konkoly Observatory, Piszkéstetô mountain, Hungary, during the nights 11-16 October 2001. The telescope was equipped with a Wright Instruments EEV CCD05$20 \mathrm{CCD}$-camera, whose pixel size of $22.5 \mu \mathrm{m}$ corresponded to 0 ' 35 on the sky. The field of view of the $756 \times 1024$ camera was $4^{\prime} \times 6^{\prime}$. Exposure times were $30-120 \mathrm{~s}$ in the $V$ and $10-60 \mathrm{~s}$ in the $I_{\mathrm{C}}$ band, depending on the brightness of the stars. Each star was observed at least twice during the two photometric nights. Data reduction including bias subtraction, flat fielding and cosmic ray removal was performed in IRAF. The $V$ and $I_{\mathrm{C}}$ magnitudes of the target stars were determined by aperture photometry using the IRAF task PHOT. In order to calibrate the magnitude scale each night we observed several times the open cluster NGC 7790, whose standard $U, B, V, R_{\mathrm{c}}$ and $I_{\mathrm{C}}$ 
Table 1. X-ray data for the observed candidates.

\begin{tabular}{|c|c|c|c|c|c|c|c|c|c|c|c|}
\hline \multirow[t]{2}{*}{ No. } & \multirow{2}{*}{$\begin{array}{l}1 \mathrm{RX} \\
\text { name }\end{array}$} & \multicolumn{2}{|c|}{ X-ray position } & \multirow[t]{2}{*}{$M L$} & \multirow{2}{*}{$\begin{array}{c}C R \\
{[\mathrm{cps}]}\end{array}$} & \multirow{2}{*}{$\begin{array}{c}\text { error } C R \\
{[\mathrm{cps}]}\end{array}$} & \multirow[t]{2}{*}{ HR1 } & \multirow{2}{*}{$\begin{array}{l}\text { error } \\
\text { HR1 }\end{array}$} & \multirow[t]{2}{*}{ HR2 } & \multirow{2}{*}{$\begin{array}{l}\text { error } \\
\text { HR2 }\end{array}$} & \multirow{2}{*}{$\begin{array}{c}f_{\mathrm{X}} / 10^{-13} \\
{\left[\mathrm{erg} \mathrm{cm} \mathrm{cm}^{-2} \mathrm{~s}^{-1}\right]}\end{array}$} \\
\hline & & RA (2000) & Dec (2000) & & & & & & & & \\
\hline 1 & J000002.5+733942 & 00:00:02.5 & $+73: 39: 42$ & 11.7 & 0.018 & 0.006 & 1 & 0.45 & 0.82 & 0.59 & 2.46 \\
\hline 2 & J000142.0+773057 & 00:01:42.0 & $+77: 30: 57$ & 47.7 & 0.029 & 0.006 & 0.85 & 0.16 & 0.47 & 0.21 & 3.74 \\
\hline 3 & J002827.8+785750 & $00: 28: 27.8$ & $+78: 57: 51$ & 32.7 & 0.041 & 0.009 & 0.36 & 0.21 & 0.29 & 0.25 & 4.22 \\
\hline 4 & J003804.2+790328 & $00: 38: 04.2$ & $+79: 03: 29$ & 161.4 & 0.110 & 0.014 & 0.06 & 0.12 & 0.07 & 0.17 & 9.49 \\
\hline 5 & J003904.2+791912 & 00:39:04.2 & $+79: 19: 13$ & 185.5 & 0.127 & 0.015 & 0.15 & 0.11 & 0.09 & 0.14 & 11.56 \\
\hline 6 & J004621.1+741251 & $00: 46: 21.1$ & $+74: 12: 51$ & 33.3 & 0.026 & 0.007 & 1 & 0.19 & 0.27 & 0.24 & 3.55 \\
\hline 7 & J005300.8+682125 & 00:53:00.8 & $+68: 21: 25$ & 56.1 & 0.065 & 0.012 & 0.22 & 0.17 & 0.12 & 0.21 & 6.16 \\
\hline 8 & $\mathrm{~J} 010117.1+713113$ & 01:01:17.1 & $+71: 31: 14$ & 42.5 & 0.039 & 0.009 & 0.66 & 0.21 & 0.31 & 0.22 & 4.65 \\
\hline 9 & J010929.0+683915 & 01:09:29.0 & $+68: 39: 16$ & 58.8 & 0.046 & 0.010 & 0.94 & 0.17 & 0.3 & 0.21 & 6.05 \\
\hline 10 & J011934.6+713027 & 01:19:34.6 & $+71: 30: 28$ & 9.7 & 0.009 & 0.005 & 1 & 0.24 & 0.68 & 0.45 & 1.24 \\
\hline 11 & J012927.4+744448 & $01: 29: 27.4$ & $+74: 44: 49$ & 53.0 & 0.039 & 0.008 & 0.44 & 0.21 & 0.25 & 0.23 & 4.19 \\
\hline 12 & J013925.5+701853 & $01: 39: 25.5$ & $+70: 18: 54$ & 86.3 & 0.056 & 0.010 & 0.98 & 0.18 & 0.02 & 0.18 & 7.58 \\
\hline 13 & J022036.3+774419 & $02: 20: 36.3$ & $+77: 44: 20$ & 11.8 & 0.016 & 0.007 & 0.65 & 0.58 & 0.68 & 0.31 & 1.83 \\
\hline 14 & J022613.0+681127 & $02: 26: 13.0$ & $+68: 11: 28$ & 30.9 & 0.032 & 0.008 & 1 & 0.24 & 0.25 & 0.25 & 4.33 \\
\hline 15 & $\mathrm{~J} 200549.1+770550$ & $20: 05: 49.1$ & $+77: 05: 50$ & 22.8 & 0.016 & 0.004 & 1 & 0.26 & 0.34 & 0.25 & 2.20 \\
\hline 16 & J201151.4+783434 & 20:11:51.4 & $+78: 34: 34$ & 43.1 & 0.023 & 0.005 & 0.88 & 0.15 & 0.07 & 0.21 & 2.96 \\
\hline 17 & $\mathrm{~J} 201424.6+660521$ & $20: 14: 24.6$ & $+66: 05: 21$ & 36.6 & 0.027 & 0.005 & 0.19 & 0.21 & 0.36 & 0.27 & 2.48 \\
\hline 18 & J201948.8+653408 & $20: 19: 48.8$ & $+65: 34: 08$ & 46.1 & 0.026 & 0.005 & 0.79 & 0.15 & 0.08 & 0.21 & 3.25 \\
\hline 19 & $\mathrm{~J} 202026.6+780717$ & $20: 20: 26.6$ & $+78: 07: 17$ & 53.2 & 0.035 & 0.006 & 0.14 & 0.17 & 0.46 & 0.19 & 3.18 \\
\hline 20 & $\mathrm{~J} 202513.8+733638$ & $20: 25: 13.8$ & $+73: 36: 38$ & 350.2 & 0.188 & 0.016 & 0.2 & 0.08 & 0.12 & 0.10 & 17.62 \\
\hline 21 & J203341.8+705321 & $20: 33: 41.8$ & $+70: 53: 21$ & 21.9 & 0.021 & 0.006 & 0.15 & 0.23 & 0.27 & 0.29 & 1.95 \\
\hline 22 & J203813.1+692413 & $20: 38: 13.1$ & $+69: 24: 14$ & 33.9 & 0.032 & 0.007 & 0.51 & 0.19 & 0.24 & 0.25 & 3.51 \\
\hline 23 & J204327.6+711546 & $20: 43: 27.6$ & $+71: 15: 47$ & 9.8 & 0.007 & 0.004 & 1 & 0.72 & 0.39 & 0.51 & 0.91 \\
\hline 24 & $\mathrm{~J} 205312.2+792031$ & $20: 53: 12.2$ & $+79: 20: 32$ & 10.5 & 0.010 & 0.004 & 0.35 & 0.41 & 0.26 & 0.38 & 0.97 \\
\hline 25 & J205607.9+651033 & 20:56:07.9 & $+65: 10: 33$ & 41.0 & 0.031 & 0.007 & 0.7 & 0.17 & 0.13 & 0.23 & 3.70 \\
\hline 26 & $\mathrm{~J} 210419.0+750359$ & 21:04:19.0 & $+75: 03: 59$ & 80.8 & 0.055 & 0.009 & 0.15 & 0.16 & 0.15 & 0.18 & 5.04 \\
\hline 27 & $\mathrm{~J} 211024.8+704600$ & $21: 10: 24.8$ & $+70: 46: 01$ & 186.2 & 0.101 & 0.012 & 0.05 & 0.11 & 0.05 & 0.15 & 8.66 \\
\hline 28 & $\mathrm{~J} 211127.5+761435$ & $21: 11: 27.5$ & $+76: 14: 35$ & 66.7 & 0.054 & 0.009 & 0.44 & 0.17 & 0.38 & 0.17 & 5.75 \\
\hline 29 & $\mathrm{~J} 211143.9+685428$ & $21: 11: 43.9$ & $+68: 54: 29$ & 22.9 & 0.016 & 0.005 & 0.49 & 0.35 & 0.69 & 0.24 & 1.76 \\
\hline 30 & $\mathrm{~J} 213253.9+703751$ & $21: 32: 53.9$ & $+70: 37: 52$ & 21.4 & 0.021 & 0.007 & 1 & 0.25 & 0.33 & 0.29 & 2.91 \\
\hline 31 & $\mathrm{~J} 213749.2+803228$ & $21: 37: 49.2$ & $+80: 32: 28$ & 91.8 & 0.056 & 0.009 & 0.61 & 0.14 & 0.24 & 0.15 & 6.50 \\
\hline 32 & $\mathrm{~J} 214258.6+803021$ & $21: 42: 58.6$ & $+80: 30: 22$ & 15.5 & 0.013 & 0.005 & 1 & 0.65 & 0.35 & 0.32 & 1.71 \\
\hline 33 & $\mathrm{~J} 220524.6+655219$ & $22: 05: 24.6$ & $+65: 52: 20$ & 48.2 & 0.054 & 0.011 & 0.76 & 0.15 & 0.18 & 0.21 & 6.61 \\
\hline 34 & J221107.9+791801 & $22: 11: 07.9$ & $+79: 18: 01$ & 72.0 & 0.047 & 0.008 & 0.11 & 0.15 & 0.13 & 0.20 & 4.21 \\
\hline 35 & $\mathrm{~J} 221158.7+681953$ & $22: 11: 58.7$ & $+68: 19: 53$ & 61.8 & 0.060 & 0.011 & 0.1 & 0.19 & 0.14 & 0.26 & 5.34 \\
\hline 36 & $\mathrm{~J} 222706.6+652127$ & $22: 27: 06.6$ & $+65: 21: 27$ & 32.9 & 0.030 & 0.008 & 0.94 & 0.20 & 0.08 & 0.25 & 4.04 \\
\hline 37 & $\mathbf{J} 223345.1+703321$ & $22: 33: 45.1$ & $+70: 33: 21$ & 35.3 & 0.036 & 0.010 & 0.82 & 0.21 & 0.77 & 0.21 & 4.49 \\
\hline 38 & $\mathrm{~J} 223956.5+774934$ & $22: 39: 56.5$ & $+77: 49: 35$ & 53.1 & 0.043 & 0.008 & 0.17 & 0.17 & 0.05 & 0.22 & 3.96 \\
\hline 39 & $\mathrm{~J} 225133.9+763549$ & $22: 51: 33.9$ & $+76: 35: 50$ & 77.5 & 0.058 & 0.009 & 0.55 & 0.15 & 0.26 & 0.17 & 6.51 \\
\hline 40 & $\mathrm{~J} 230043.2+772833$ & $23: 00: 43.2$ & $+77: 28: 33$ & 166.6 & 0.098 & 0.012 & 0.23 & 0.11 & 0.31 & 0.14 & 9.32 \\
\hline 41 & $\mathrm{~J} 230533.0+782238$ & 23:05:33.0 & $+78: 22: 39$ & 96.6 & 0.058 & 0.009 & 0.11 & 0.14 & 0.43 & 0.17 & 5.11 \\
\hline 42 & $\mathrm{~J} 230757.5+765146$ & $23: 07: 57.5$ & $+76: 51: 47$ & 26.3 & 0.027 & 0.007 & 1 & 0.18 & 0.25 & 0.25 & 3.67 \\
\hline 43 & $\mathrm{~J} 230942.9+735725$ & 23:09:42.9 & $+73: 57: 25$ & 36.5 & 0.052 & 0.010 & 0.44 & 0.20 & 0.31 & 0.22 & 5.49 \\
\hline 44 & $\mathrm{~J} 231616.5+784156$ & $23: 16: 16.5$ & $+78: 41: 57$ & 661.7 & 0.238 & 0.016 & 0.07 & 0.07 & 0.32 & 0.09 & 20.66 \\
\hline 45 & $\mathrm{~J} 234342.3+684631$ & $23: 43: 42.3$ & $+68: 46: 32$ & 39.3 & 0.042 & 0.010 & 0.85 & 0.22 & 0.37 & 0.25 & 5.36 \\
\hline 46 & $\mathrm{~J} 235107.5+785802$ & $23: 51: 07.5$ & $+78: 58: 02$ & 102.7 & 0.069 & 0.010 & 0.16 & 0.13 & 0.28 & 0.16 & 6.28 \\
\hline
\end{tabular}


Table 2. Optical counterparts.

\begin{tabular}{|c|c|c|c|c|c|c|}
\hline \multirow[t]{2}{*}{ No. } & \multirow{2}{*}{$\begin{array}{l}\text { GSC } \\
\text { name }\end{array}$} & \multicolumn{2}{|c|}{ Optical position } & \multirow{2}{*}{$\begin{array}{c}m_{V} \\
{[\mathrm{mag}]}\end{array}$} & \multirow{2}{*}{$\begin{array}{c}\text { Separation } \\
\text { [arcsec] }\end{array}$} & \multirow{2}{*}{$\begin{array}{c}\log \\
f_{\mathrm{X}} / f_{V}\end{array}$} \\
\hline & & RA (2000) & Dec (2000) & & & \\
\hline 1 & 0430601190 & $00: 00: 02.1$ & $+73: 39: 46$ & 12.0 & 7 & -2.44 \\
\hline 2 & 0449602286 & 00:01:42.5 & $+77: 31: 06$ & 11.7 & 8 & -2.39 \\
\hline 3 & 0450000730 & $00: 28: 28.1$ & $+78: 57: 43$ & 10.4 & 12 & -2.87 \\
\hline 4 & 0450001478 & 00:38:05.4 & $+79: 03: 21$ & 9.3 & 10 & -2.95 \\
\hline 5 & 0450001549 & 00:39:06.1 & $+79: 19: 10$ & 11.9 & 8 & -1.79 \\
\hline 6 & 0430701640 & $00: 46: 22.3$ & $+74: 12: 49$ & 12.7 & 3 & -1.99 \\
\hline 7 & 0429600542 & $00: 53: 00.5$ & $+68: 21: 22$ & 12.2 & 6 & -1.98 \\
\hline 8 & 0430401407 & 01:01:17.3 & $+71: 31: 13$ & 10.6 & 3 & -2.71 \\
\hline 9 & 0429600222 & 01:09:28.8 & $+68: 39: 15$ & 10.1 & 4 & -2.81 \\
\hline 10 & 0430501893 & $01: 19: 34.2$ & $+71: 30: 33$ & 11.0 & 2 & -3.13 \\
\hline 11 & 0430900722 & $01: 29: 28.6$ & $+74: 44: 49$ & 12.8 & 4 & -1.91 \\
\hline 12 & 0431401757 & 01:39:25.9 & $+70: 18: 50$ & 9.8 & 2 & -2.82 \\
\hline 13 & 0449902076 & $02: 20: 37.2$ & $+77: 44: 13$ & 11.9 & 17 & -2.61 \\
\hline 14 & 0431200734 & $02: 26: 12.8$ & $+68: 11: 33$ & 12.3 & 9 & -2.10 \\
\hline 15 & 0458902335 & 20:05:48.9 & $+77: 05: 53$ & 10.4 & 13 & -3.11 \\
\hline 16 & 0458900369 & 20:11:53.6 & $+78: 34: 37$ & 11.1 & 10 & -2.71 \\
\hline 17 & 0424400322 & $20: 14: 26.1$ & $+66: 05: 19$ & 11.2 & 5 & -2.76 \\
\hline 18 & 0424101092 & $20: 19: 48.7$ & $+65: 34: 11$ & 10.5 & 7 & -2.92 \\
\hline 19 & 0458901101 & 20:20:29.3 & $+78: 07: 22$ & 9.9 & 4 & -3.18 \\
\hline 20 & 0445900083 & $20: 25: 15.4$ & $+73: 36: 33$ & 10.7 & 7 & -2.11 \\
\hline 21 & 0445101270 & $20: 33: 43.7$ & $+70: 53: 18$ & 11.7 & 6 & -2.66 \\
\hline 22 & 0445100172 & 20:38:13.9 & $+69: 24: 12$ & 12.8 & 6 & -1.98 \\
\hline 23 & 0445500856 & $20: 43: 28.4$ & $+71: 15: 47$ & 11.7 & 2 & -2.99 \\
\hline 24 & 0459401127 & $20: 53: 12.0$ & $+79: 20: 22$ & 11.9 & 9 & -2.88 \\
\hline 25 & 0425500314 & 20:56:09.3 & $+65: 10: 30$ & 10.3 & 7 & -2.94 \\
\hline 26 & 0458600348 & 21:04:20.7 & $+75: 04: 04$ & 11.1 & 6 & -2.48 \\
\hline 27 & 0446401372 & $21: 10: 24.5$ & $+70: 45: 58$ & 11.0 & 5 & -2.29 \\
\hline 28 & 0458601090 & 21:11:29.4 & $+76: 14: 29$ & 11.4 & 5 & -2.32 \\
\hline 29 & 0446001614 & 21:11:44.0 & $+68: 54: 22$ & 10.4 & 9 & -3.24 \\
\hline 30 & 0446501410 & $21: 32: 53.8$ & $+70: 37: 43$ & 12.0 & 7 & -2.38 \\
\hline 31 & 0460700461 & $21: 37: 47.4$ & $+80: 32: 31$ & 11.0 & 5 & -2.42 \\
\hline 32 & 0460700611 & $21: 42: 57.6$ & $+80: 30: 19$ & 11.3 & 2 & -2.87 \\
\hline 33 & 0427501998 & $22: 05: 25.8$ & $+65: 52: 19$ & 10.9 & 6 & -2.46 \\
\hline 34 & 0460801986 & 22:11:11.0 & $+79: 18: 00$ & 12.7 & 6 & -1.95 \\
\hline 35 & 0446300818 & $22: 11: 57.8$ & $+68: 19: 52$ & 11.5 & 9 & -2.30 \\
\hline 36 & 0427200174 & $22: 27: 05.3$ & $+65: 21: 31$ & 12.7 & 13 & -1.96 \\
\hline 37 & 0448000917 & $22: 33: 44.9$ & $+70: 33: 18$ & 10.7 & 9 & -2.70 \\
\hline 38 & 0460400743 & $22: 39: 58.1$ & $+77: 49: 40$ & 11.7 & 6 & -2.37 \\
\hline 39 & 0460101894 & $22: 51: 35.8$ & $+76: 35: 54$ & 11.4 & 5 & -2.26 \\
\hline 40 & 0460502733 & $23: 00: 44.4$ & $+77: 28: 38$ & 10.9 & 5 & -2.31 \\
\hline 41 & 0460500268 & $23: 05: 36.1$ & $+78: 22: 39$ & 13.0 & 8 & -1.72 \\
\hline 42 & 0460100938 & $23: 07: 56.4$ & $+76: 51: 47$ & 10.7 & 14 & -2.77 \\
\hline 43 & 0448900036 & 23:09:43.4 & $+73: 57: 15$ & 12.0 & 9 & -2.08 \\
\hline 44 & 0460500170 & $23: 16: 18.1$ & $+78: 41: 56$ & 11.8 & 2 & -1.59 \\
\hline 45 & 0447900348 & $23: 43: 41.9$ & $+68: 46: 27$ & 12.6 & 8 & -1.88 \\
\hline 46 & 0461001318 & $23: 51: 10.0$ & $+78: 58: 05$ & 11.4 & 4 & -2.27 \\
\hline
\end{tabular}




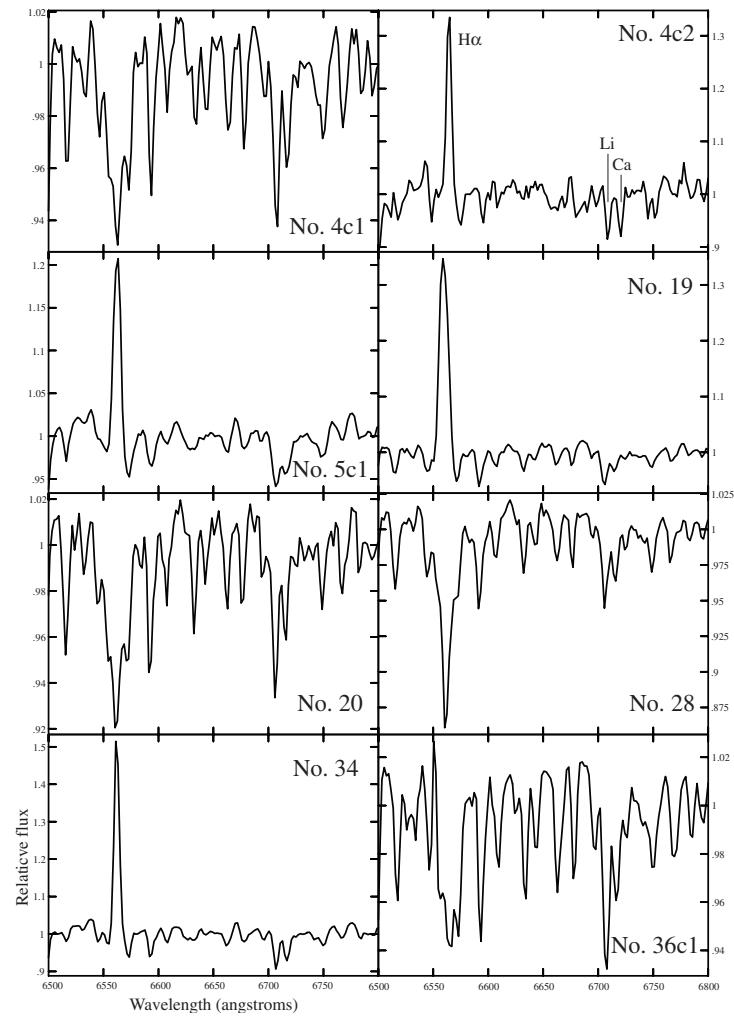

Fig. 1. Spectra for the stars that have significant Li detections.

magnitudes are given in Petrov et al. (2001). The error of the photometry is $\pm 0.04 \mathrm{mag}$.

\section{Results}

\subsection{The Li-rich stars}

Among the 46 target stars, we have detected significant Li absorption lines in 16 stars as shown in Fig. 1. The typical value of $W(\mathrm{Li})$ is $0.4 \AA$ and ranges from 0.28 to $0.46 \AA$. Note that at the spectral resolution of CAFOS, the Li I line is subject to overestimation due to blending with nearby lines. Nonetheless, all these $\mathrm{Li}$ lines are stronger than the nearby $\mathrm{Ca}$ lines and are well resolved, as seen in Fig. 1. Thus we regard these objects as good candidates for being young pre-main sequence stars. Eight stars show $\mathrm{H} \alpha$ line in emission, which is another sign of stellar youth. These equivalent widths are summarized in Table 3 together with those of $\mathrm{Na}$ I and $\mathrm{Ca}$ I.

The positions of the observed stars are plotted on the molecular cloud map observed in CO (Dame et al. 2001). The Li-rich stars shown by the filled and gray circles are distributed not only inside the molecular clouds, but also outside of them. Especially remarkable is the subset of Li-rich stars distributed over the region $115^{\circ}<l<123^{\circ}$ and $15^{\circ}<b<19^{\circ}$, where $\mathrm{CO}$ intensity is relatively weak. They are aligned parallel to the galactic plane, apparently connecting the dark clouds MBM 163-164 and LDN 1333. Note that the elongation of the filamentary cloud of LDN 1333 is nearly parallel to the alignment of the Li-rich stars. We will further discuss the distributions of the Li-rich stars and the dark clouds in Sect. 4.1.

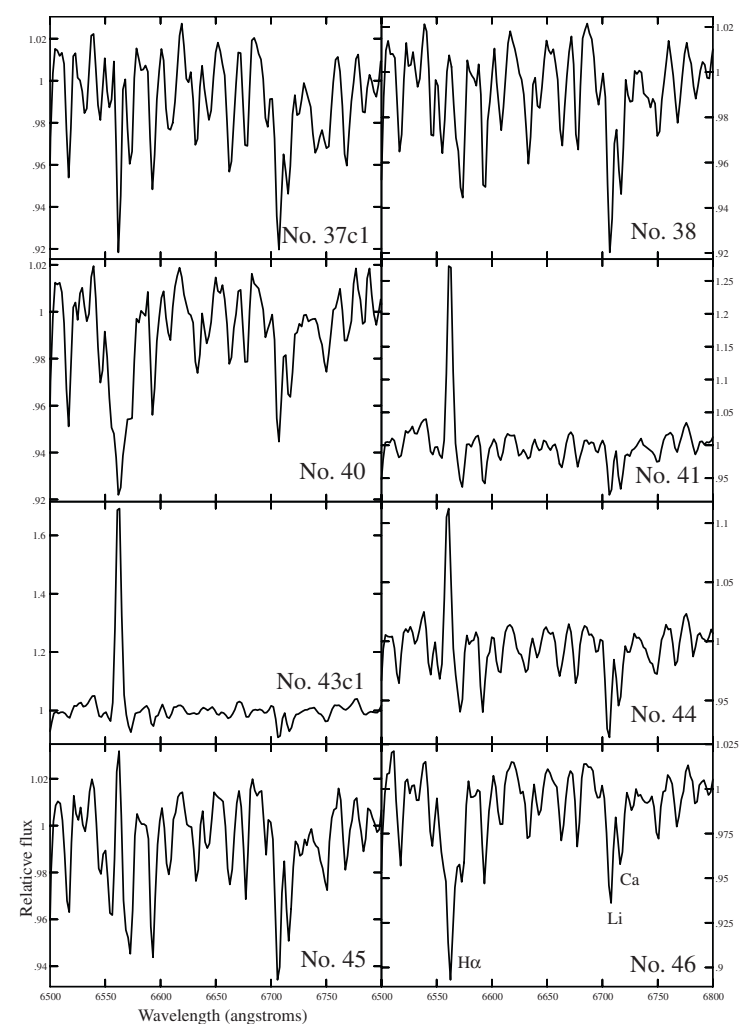

\subsection{Spectral type determination}

The $V-I_{\mathrm{C}}$ colour indices, listed in Table 3, provide a rough estimate of the spectral types if we assume our target stars to be free of interstellar reddening. As they are projected mostly outside the molecular clouds (Fig. 2) this assumption seems reasonable. The measured colour indices suggest spectral types later than K0 (Kenyon \& Hartmann 1995). For a more accurate spectral classification we studied several absorption features as follows.

The equivalent width of the Na I and Ca I lines $(\lambda=5890 \AA$ and $6161 \AA)$ are well correlated with the spectral type. We compared the $W(\mathrm{NaI})$ and $W(\mathrm{CaI})$ with those of the standard stars, which were observed with the same telescope and instrument. We also calculated several flux ratios suggested to be good indicators of spectral type. These are $I 1=F(7021-7050) / F(6960-6990)$, $I 2=F(6510-6540) / F(6370-6400), I 3=F(6510-6540) /$ $F(6660-6690)$ (Martin \& Kun 1996), $B=F(7375-7385) /$ $F(7353-7363)$ (Kirkpatrick et al. 1991), and $T 1=$ $F(7061-7088) / F(7024-7055), T 2=F(7095-7126) /$ $F$ (7024-7055) (Preibisch et al. 2001) where $F$ denotes the flux integrated over the wavelength range in angstroms. These indices show linear correlations with spectral type for the standard stars and their fitted correlation functions were applied to the target stars. Each spectral feature resulted in a spectral type and their averages are listed in Table 3. The uncertainty of the spectral classification is \pm 1 spectral subclasses. In most cases the spectral types determined by the line equivalent widths and the spectral indices agree well with those by the $V-I_{\mathrm{C}}$ colours, suggesting that most of our 
Table 3. Observed parameters of the new T Tauri stars.

\begin{tabular}{|c|c|c|c|c|c|c|c|c|c|c|c|c|}
\hline No & $\begin{array}{l}\text { GSC } \\
\text { name }\end{array}$ & $\begin{array}{c}W(\mathrm{H} \alpha) \\
(5653)[\AA]\end{array}$ & $\begin{array}{c}W(\mathrm{Li})^{1} \\
(6708)[\AA]\end{array}$ & $\begin{array}{c}W(\mathrm{NaI}) \\
(5890)[\AA]\end{array}$ & $\begin{array}{c}W(\mathrm{Ca} \mathrm{I}) \\
(6161)[\AA]\end{array}$ & $\begin{array}{c}V \\
{[\mathrm{mag}]}\end{array}$ & $\begin{array}{c}I_{\mathrm{C}} \\
{[\mathrm{mag}]}\end{array}$ & $\begin{array}{l}V-I_{\mathrm{C}} \\
{[\mathrm{mag}]}\end{array}$ & $\begin{array}{c}\mathrm{Sp} \\
\text { types }\end{array}$ & $\begin{array}{c}m_{\mathrm{bol}} \\
{[\mathrm{mag}]}\end{array}$ & $\begin{array}{c}\log L_{\text {bol }} \\
{\left[L_{\odot}\right]} \\
\end{array}$ & $\begin{array}{c}\log \\
L_{\mathrm{X}} / L_{\mathrm{bol}} \\
\end{array}$ \\
\hline $4 \mathrm{c} 1$ & 0450001478 & 0.7 & 0.4 & 2.5 & 1.1 & 10.43 & 9.50 & 0.92 & K1 & 10.06 & 0.48 & -3.41 \\
\hline $4 c 2$ & & -1.9 & 0.4 & 6.0 & 2.2 & 13.86 & 12.09 & 1.77 & K7 & 12.94 & -0.68 & -2.26 \\
\hline $5 \mathrm{c} 1$ & 0450001549 & -1.6 & 0.5 & 7.4 & 3.0 & 12.18 & 10.66 & 1.52 & K6 & 11.36 & -0.04 & -2.80 \\
\hline $5 \mathrm{c} 2$ & & & & & & 14.12 & 12.03 & 2.10 & $\mathrm{M} 2^{2}$ & 12.48 & -0.49 & -2.35 \\
\hline 19 & 0458901101 & -3.4 & 0.4 & 1.5 & 0.8 & 10.39 & 9.57 & 0.82 & G8 & 10.14 & 0.44 & -3.85 \\
\hline 20 & 0445900083 & 0.9 & 0.4 & 1.5 & 0.8 & 10.62 & 9.78 & 0.84 & K0 & 10.31 & 0.37 & -3.04 \\
\hline 28 & 0458601090 & 1.2 & 0.3 & 1.5 & 0.8 & 11.66 & 10.85 & 0.81 & G8 & 11.41 & -0.06 & -3.09 \\
\hline 34 & 0460801986 & -3.0 & 0.5 & 5.6 & 2.1 & 13.09 & 11.53 & 1.55 & K7 & 12.17 & -0.37 & -2.92 \\
\hline $36 \mathrm{c} 1$ & 0427200174 & 0.9 & 0.5 & 2.0 & 1.1 & 12.92 & 11.79 & 1.13 & K4 & 12.37 & -0.45 & -2.85 \\
\hline $36 \mathrm{c} 2$ & & & & & & 14.69 & 11.85 & 2.84 & $\mathrm{M} 4^{2}$ & 12.13 & -0.36 & -2.95 \\
\hline $37 \mathrm{c} 1$ & 0448000917 & 0.4 & 0.6 & 2.1 & 0.8 & 11.63 & 9.98 & 1.65 & $\mathrm{~K} 3$ & $9.70^{4}$ & 0.62 & -3.88 \\
\hline $37 \mathrm{c} 2$ & & & & & & 14.86 & 13.75 & 1.12 & $\mathrm{~F} 4^{3}$ & $13.30^{4}$ & -0.82 & -2.43 \\
\hline 38 & 0460400743 & 0.0 & 0.5 & 2.2 & 1.2 & 11.88 & 10.86 & 1.03 & $\mathrm{~K} 2$ & 11.46 & -0.09 & -3.22 \\
\hline 40 & 0460502733 & 1.2 & 0.4 & 1.6 & 1.0 & 10.98 & 10.10 & 0.88 & K0 & 10.67 & 0.23 & -3.17 \\
\hline 41 & 0460500268 & -1.8 & 0.4 & 4.4 & 2.1 & 13.19 & 11.60 & 1.59 & K6 & 12.37 & -0.45 & -2.75 \\
\hline $43 \mathrm{c} 1$ & 0448900036 & -4.3 & 0.5 & 5.5 & 2.8 & 13.21 & 11.35 & 1.86 & K7 & 12.29 & -0.42 & -2.75 \\
\hline $43 c 2$ & & & & & & 15.55 & 13.05 & 2.50 & $\mathrm{M} 3^{2}$ & 13.52 & -0.91 & -2.26 \\
\hline 44 & 0460500170 & -0.6 & 0.5 & 3.9 & 1.6 & 11.77 & 10.54 & 1.24 & $\mathrm{~K} 4$ & 11.22 & 0.01 & -2.60 \\
\hline 45 & 0447900348 & -0.1 & 0.4 & 1.9 & 1.3 & 12.64 & 11.64 & 1.00 & $\mathrm{~K} 2$ & 12.22 & -0.39 & -2.79 \\
\hline 46 & 0461001318 & 0.9 & 0.4 & 1.9 & 1.0 & 11.34 & 10.41 & 0.93 & $\mathrm{~K} 1$ & 10.97 & 0.11 & -3.22 \\
\hline
\end{tabular}

${ }^{1}$ Up to $0.14 \AA$ A have to be subtracted due to blending with Fe lines.

${ }^{2}$ Derived only from the $V-I_{\mathrm{C}}$ colours.

${ }^{3}$ Derived from the $V-I_{\mathrm{C}}$ colour and the reddening of $E\left(V-I_{\mathrm{C}}\right)=0.57$ is assumed as estimated from $37 \mathrm{c} 1$.

${ }^{4}$ Extinction of $A_{\mathrm{V}}=1.43$ is applied.

stars are free of reddening. The apparent exception is $37 \mathrm{c} 1$ whose $V-I_{\mathrm{C}}$ colour index, compared the spectral type derived from $W(\mathrm{NaI})$ and $W(\mathrm{CaI})$ equivalent widths and flux ratios, indicates a reddening $E\left(V-I_{\mathrm{C}}\right)=0.57$, corresponding to the visual extinction $A_{\mathrm{V}}=1.43 \mathrm{mag}$ (Cohen et al. 1981). These reddening and extinction values are also applied for the nearby star 37c2. Spectral types of companion candidates whose spectra were not observed were estimated from the $V-I_{\mathrm{C}}$ colour indices.

\subsection{HR diagram and physical parameters of the PMS stars}

Because $W(\mathrm{Li})$ is a function of age and spectral type, it is compared with the values measured in Pleiades, IC 2602, and Taurus, whose ages are estimated to be $\sim 10^{8} \mathrm{yr}, \sim 3 \times 10^{7} \mathrm{yr}$, and a few $\times 10^{6} \mathrm{yr}$, respectively (Fig. 3). It can clearly be seen that the Li-rich stars in the Cep-Cas region have significantly larger $W(\mathrm{Li})$ than the Pleiades and IC 2602 star of the same spectral types. However, our $W(\mathrm{Li})$ can be overestimated due to the low spectral resolution. There are Fe I lines at $6703.5 \AA$, $6705.1 \AA$, $6707.4 \AA$ and $6710.3 \AA$ that may be blended with the Li line. For K-type stars the total equivalent width of these Fe I lines is
$0.14 \pm 0.04 \AA$ (Zboril et al. 1997). When we take into account the overestimation for $W(\mathrm{Li})$, the points in Fig. 3 decrease, and stars $4 \mathrm{c} 1,28,40,45$ and 46 are below the upper envelope of the Pleiades stars and around the same positions as stars in IC 2602. These weak Li stars (marked by gray circles in Fig. 2) cannot be young PMS stars, but instead post-TTSs or zero-age main sequence stars. Nevertheless, these boundaries seem to be ambiguous since several Taurus TTSs whose spectra were taken with high-resolution spectrographs have slightly weaker Li lines than the boundary. The spread in Li strength at any given age and spectral type range is also due to different rotation. Another possibility for these weak Li stars is active binary stars, which have excess X-ray luminosity and occasionally enhanced Li lines, as shown by Torres et al. (2002), but their sample selection is different and their stars all show less lithium. It should be noted that half of our Li-rich stars show $\mathrm{H} \alpha$ and Xray emission, which are another indication of youth, while no IC 2602 stars do so. Thus the Li-rich stars, particularly those with strong $\mathrm{Li}$ absorption or $\mathrm{H} \alpha$ emission lines, are likely to be young PMS stars. For more accurate $W(\mathrm{Li})$ estimation and verification of binarity, high resolution spectroscopy is needed.

In order to estimate the age and mass of the Li-rich stars, we plotted them on the HR diagram (Fig. 4) together with the 


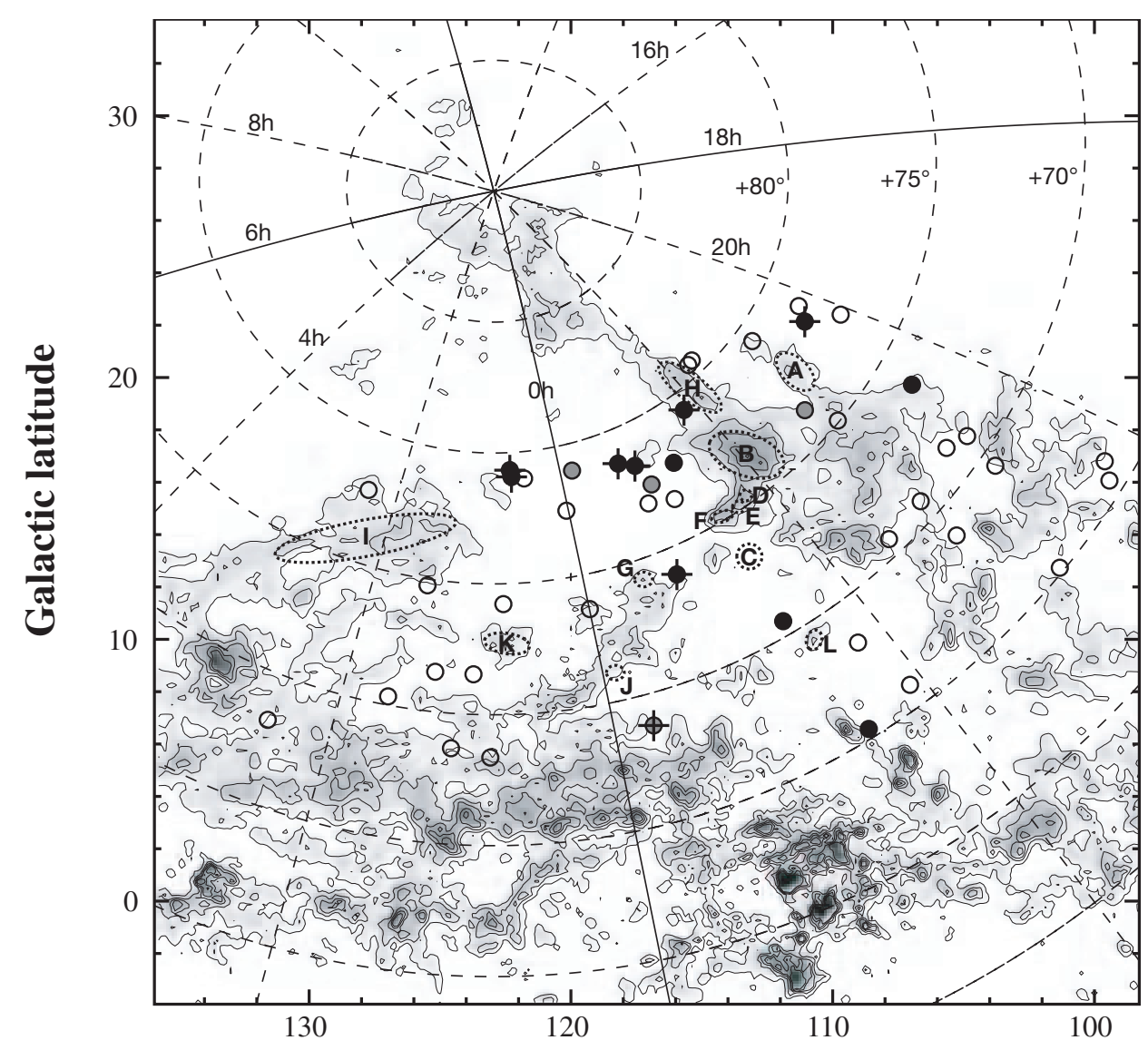

Galactic longitude

Fig. 2. The observed stars shown on the CO integrated intensity map (Dame et al. 2001) in Galactic coordinates. The equatorial gird is also drawn with dashed lines. Contours are from $3 \mathrm{~K} \mathrm{~km} \mathrm{~s}^{-1}$ with intervals of $6 \mathrm{~K} \mathrm{~km} \mathrm{~s}^{-1}$. The filled, gray and open circles denote observed X-ray point sources whose spectra are strong, weak, and without Li detection, respectively (see Sect. 3.3). The crosses are those with $\mathrm{H} \alpha$ lines in emission. The dotted ellipses show the nearby dark clouds of (A) LDN 1228, (B) LDN 1241, (C) LDN 1242, (D) LDN 1243, (E) LDN 1247, (F) LDN 1251, (G) LDN 1259-1262, (H) MBM 163-165, (I) LDN 1333, (J) LDN 1274, (K) Khavtassi 15, and (L) LDN 1221 around the CO void (see text).

theoretical isochrones and evolutionary tracks by D'Antona \& Mazzitelli (1994). Because young stars are expected to exist close to molecular clouds, we adopted a distance of $200 \mathrm{pc}$ which is the smallest distance to the clouds around the Li-rich stars. The $L_{\text {bol }}$ may be larger in reality if they are at the same distance as farther clouds. Nonetheless, most of the target stars are distributed above the main sequence and are likely to be PMS stars. Note that the binary-pair candidates 5c1-c2 and $43 \mathrm{c} 1-\mathrm{c} 2$ are placed on the same isochrones, while $4 \mathrm{c} 1-\mathrm{c} 2$ and $36 \mathrm{c} 1-\mathrm{c} 2$ are mismatched. The star $37 \mathrm{c} 2$ is below the main sequence while $37 \mathrm{c} 1$ is in the PMS area. It seems that $37 \mathrm{c} 2$ is a reddened background star. Taking into account the uncertainty of the spectral classification and photometry, the age difference between 4c1-c2 and 36c1-c2 may be smaller, and thus can be reconciled with the binary nature of these stars.

The estimated masses and ages of the PMS candidates are listed in Table 4. Fifteen out of the 19 PMS candidates (excluding the apparent background star 37c2) are younger than $10 \mathrm{Myr}$ if their distances are larger than $200 \mathrm{pc}$. If the real distance is about a half of the assumed value and they are not related to the molecular clouds, the luminosity at $10 \mathrm{Myr}$ star drops down to the main sequence. However, only a small number of young star clusters have been found within $100 \mathrm{pc}$ of the sun. Because of being bright in X-ray and Li-richness, they are not likely to be main sequence stars, but young PMS stars. Under the assumption of $d=200 \mathrm{pc}$, the estimated masses are $0.4 M_{\odot}<M<2 M_{\odot}$, hence they are T Tauri stars.

\section{Discussion}

\subsection{Distribution of the T Tauri stars}

As mentioned in Sect. 3.1 the TTS are not uniformly distributed, but seven of them $(4,5,38,40,41,44,46)$ are grouped around $115^{\circ} \lesssim l \lesssim 123^{\circ}$ and $b \sim 16^{\circ}$, where almost no $\mathrm{CO}$ clouds are found. The group of TTSs are hence separated from the CO clouds, like those often referred to as "isolated TTSs", which have been found in many SFRs (e.g., Neuhäuser et al. 1997). In this section, we discuss the physical associations of the TTSs with their possible parental molecular clouds.

There are several dark clouds around the $\mathrm{CO}$ void whose distances are estimated to be from 180 pc to 300 pc by Kun (1998) and Obayashi et al. (1998). The TTSs discussed here 


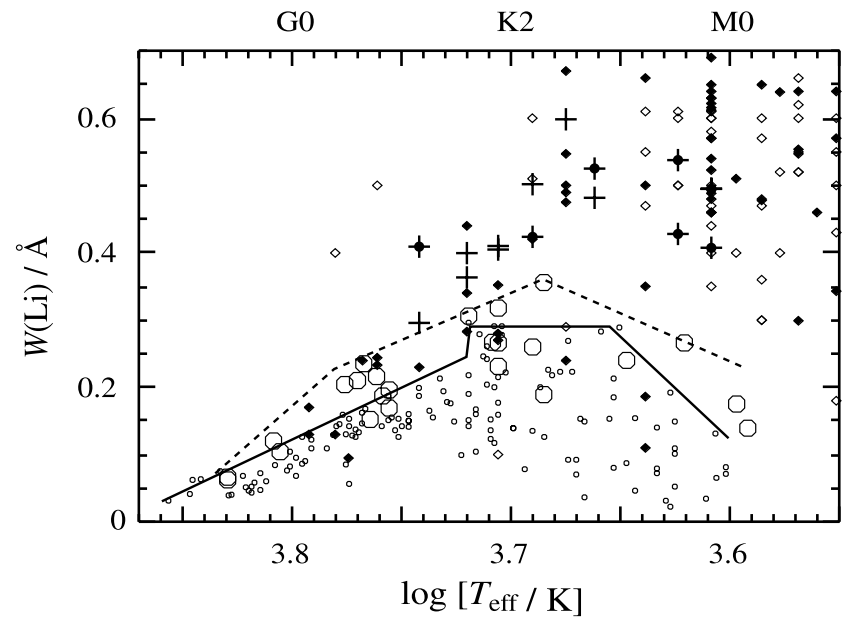

Fig. 3. Li equivalent width versus effective temperature. The Li-rich stars detected in the Cep-Cas region are shown by the crosses, and those with $\mathrm{H} \alpha$ emission lines are by black dots. The small open circles and the large circles are Pleiades and IC 2602 stars, respectively, whose upper envelopes are drawn with the solid and the dashed lines (Neuhäuser 1997; Neuhäuser et al. 1997, and the references therein). The $\mathrm{T}$ Tauri stars in Taurus whose $W(\mathrm{Li})$ were measured by lowresolution and high-resolution spectrograph are also shown by open and filled diamonds, respectively.

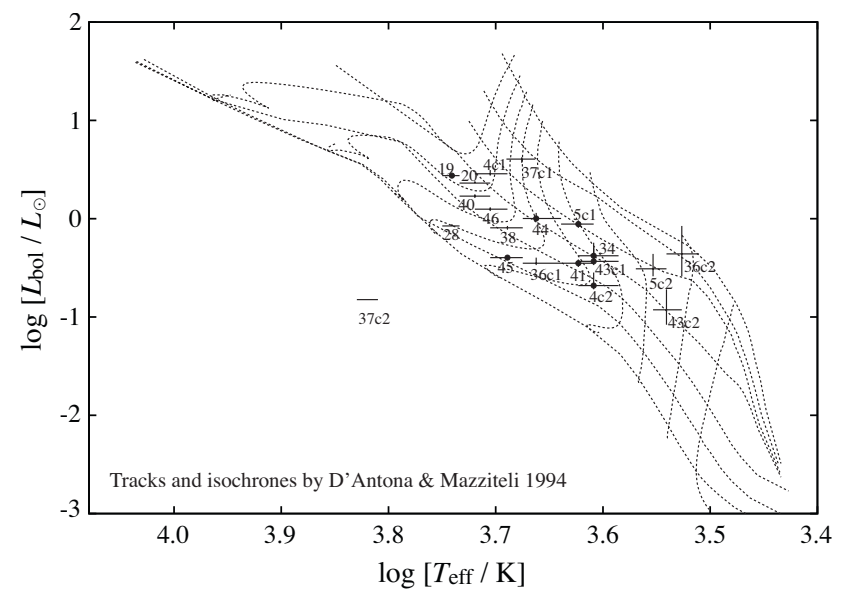

Fig. 4. HR diagram for the PMS candidates. Tracks are for 0.1, 0.2, $0.4,0.6,0.8,1,1.2,1.5$ and $2 M_{\odot}$, and isochrones are for 0.1, 0.3, 1, 3, 10, 30 and $100 \mathrm{Myr}$, both taken from D'Antona \& Mazzitelli (1994). Stars are assumed to be located in the Cep-Cas SFR at $200 \mathrm{pc}$. The error bars are drawn according to the spectral classification uncertainty of \pm 1 subclass. The stars with the $\mathrm{H} \alpha$ emission lines are marked with black dots.

are projected inside the void located between the dark clouds of LDN $1333\left(l=128.81, b=13^{\circ} .68, d=180 \mathrm{pc}\right.$, mark I in Fig. 2) and $\operatorname{LDN} 1241\left(l=113^{\circ} .03, b=17.51, d=300 \mathrm{pc}\right.$, mark B in Fig. 2), and appear to form a connection between these two clods. The distances of these clouds are different. However, in the Cepheus flare region above $b \gtrsim+17^{\circ}$ two overlapping cloud layers were found by Kun (1998, see her Fig. 5d), whose distances are 200 and 300 pc, respectively. The 200 pc components, including LDN 1228 (mark A in Fig. 2) and MBM 163-165 (mark H in Fig. 2), dominate at higher galactic latitudes, and both layers can be recognized
Table 4. Mass and age of the new T Tauri stars.

\begin{tabular}{cccc}
\hline \hline No & $\begin{array}{c}\text { GSC } \\
\text { name }\end{array}$ & $\begin{array}{c}\text { Mass } \\
\left(M_{\odot}\right)\end{array}$ & $\begin{array}{c}\text { Age } \\
(\mathrm{Myr})\end{array}$ \\
\hline $4 \mathrm{c} 1$ & 0450001478 & 1.6 & 2 \\
$4 \mathrm{c} 2$ & & 0.8 & 15 \\
$5 \mathrm{c} 1$ & 0450001549 & 0.6 & 1 \\
$5 \mathrm{c} 2$ & & 0.4 & 1 \\
19 & 0458901101 & 1.6 & 6 \\
20 & 0445900083 & 1.6 & 4 \\
28 & 0458601090 & 1.0 & 25 \\
34 & 0460801986 & 0.7 & 3 \\
$36 \mathrm{c} 1$ & 0427200174 & 0.9 & 20 \\
$36 \mathrm{c} 2$ & & 0.2 & 0.2 \\
$37 \mathrm{c} 1$ & 0448000917 & 1.0 & 0.4 \\
38 & 0460400743 & 1.2 & 8 \\
40 & 0460502733 & 1.4 & 6 \\
41 & 0460500268 & 0.8 & 7 \\
$43 \mathrm{c} 1$ & 0448900036 & 0.3 & 4 \\
$43 \mathrm{c} 2$ & & 0.7 & 3 \\
44 & 0460500170 & 1.0 & 2 \\
45 & 0447900348 & 0.9 & 25 \\
46 & 0461001318 & 1.3 & 6 \\
\hline
\end{tabular}

towards LDN 1241. Moreover, LDN 1241, MBM 163-165, and LDN 1333 were reported to have multiple velocity components (Yonekura et al. 1997; Obayashi et al. 1998). This may imply that not only LDN 1241 but also the others have multiple distance components overlapping on the same line of sight. No star formation signposts have been identified in LDN 1241. If this cloud and the group of TTSs to the northwest of it were related to each other, we would expect to see the spread of the star formation into the cloud, traced by embedded sources and outflows. It is more likely that the TTSs are not related to LDN 1241, but are foreground objects.

Several small molecular clouds are projected within the $\mathrm{CO}$ void, and most of them are reported to be closer to us than the main body of the Cepheus flare complex, namely, LDN 12591261 (mark G in Fig. 2) at $180 \pm 30$ pc (Kun 1998), LDN 1274 (mark J in Fig. 2) at $200 \pm 30$ pc (Nikolić et al. 2001) and Khavtassi 15 (mark K in Fig. 2) at 250 25 pc (Kiss et al. 2000), and these are all detected in ${ }^{13} \mathrm{CO}$ as well. The projected separations from the TTSs to the nearby ${ }^{13} \mathrm{CO}$ clouds including LDN 1333 and MBM 163-165 are estimated to be $\gtrsim 3^{\circ}$ which corresponds to $\gtrsim 10 \mathrm{pc}$ at the distance of $200 \mathrm{pc}$. In the Chamaeleon region, Mizuno et al. (1998) reported that small and dense clouds were found in ${ }^{13} \mathrm{CO}$ close to the the $\mathrm{X}$-ray emitting isolated TTSs. The typical mass of the small ${ }^{13} \mathrm{CO}$ clouds is $\sim 20 M_{\odot}$, which is comparable to those in the CepCas region. However, most of the TTSs in Chamaeleon are distributed within 4 pc from the clouds, much smaller than our results.

Apparently there is a loose complex of small and faint clouds in the same area where our $\mathrm{T}$ Tauri stars are found. However, these clouds are not detected in ${ }^{13} \mathrm{CO}$ (Yonekura et al. 1997), and thus have low density, although no sensitive ${ }^{13} \mathrm{CO}$ survey has been done as in Chamaeleon. For example, 
the stars $4 \mathrm{c} 1$ (2 Myr), 4c2 (15 Myr) and 5c1 (1 Myr) are close to a faint, low density cloud and another faint cloud is also near the stars 41 (7 Myr) and 44 (2 Myr), while the star 46 (6 Myr) is separated from the faint clouds by $\sim 2.5^{\circ}$, although $4 \mathrm{c} 1$ and 46 are weak Li stars. The mass of these faint clouds are each estimated to be $\sim 100 M_{\odot}$ from the $\mathrm{CO}$ intensity. These faint clouds are likely to be associated with the nearby 4 TTSs, however, the clouds are too low-mass to be responsible for all the TTSs inside the CO void. The total mass of the group of TTSs is estimated to be $\sim 8.8 M_{\odot}$, which yields a star formation efficiency (SFE) as high as $4 \%$ if the majority of the $\mathrm{CO}$ gas still remains after the formation of the TTSs. This value is significantly higher than that of typical nearby SFRs (Tachihara et al. 2002). Note that the SFE is molecular tracer-dependent and that the cloud mass traced by ${ }^{13} \mathrm{CO}$ is typically $\sim 60 \%$ of that by ${ }^{12} \mathrm{CO}$ (Mizuno et al. 1998, 2001). In addition, the ${ }^{12} \mathrm{CO}$ survey in Chamaeleon (Mizuno et al. 2001) detected diffuse cloud envelopes that connect the small ${ }^{13} \mathrm{CO}$ clouds to the large main clouds and surrounds many of the X-ray emitting TTSs except for the $\eta$ Cha cluster. More than $65 \%$ of the TTSs are located within $1 \mathrm{pc}$ of the cloud boundary. Compared with these results in Chamaeleon, the TTSs in the Cep-Cas void are obviously more isolated from the clouds even though the sensitivity of the CO survey is not as high as in Chamaeleon.

\subsection{Origin of the isolated $T$ Tauri stars}

The origin of isolated TTSs has been discussed by many authors (e.g. Tachihara et al. 2001). Two theories were suggested; (1) the isolated TTSs were ejected from the parent clouds by the dynamical encounters with high space velocity as run-away TTSs (ejection model; Sterzik et al. 1995) or (2) they were formed in relatively small clouds which were dissipated soon after star formation (in situ model; Feigelson 1996). Recent observational results favor the in situ model (e.g., Mizuno et al. 1998). Assuming that the TTSs have a proper motion of $1 \mathrm{~km} \mathrm{~s}^{-1}$ with respect to the clouds, they can move only $5 \mathrm{pc}$ in $5 \mathrm{Myr}$ from their birth places, slightly less than the separations for the present TTSs with the given ages. In addition, the present TTSs of a few Myr age are still in a loose group. These facts suggest that the in situ model is more plausible for the formation history of the isolated TTSs. In general, cloud dissipation is more efficient near OB stars due to the strong UV radiation, stellar winds and supernova explosions, and the projected cloud-to-TTS separations are significantly larger than in regions without OB stars (Tachihara et al. 2001). The TTSs in the Cep-Cas void are, however, considerably isolated without any nearby OB stars. Although the X-ray emitting TTSs in Chamaeleon are slightly younger than those in Cep-Cas (Alcalá et al. 1997), the cloud-to-TTS separations are not different between the TTSs younger than $5 \mathrm{Myr}$ and those older in Chamaeleon (Tachihara et al. 2001). This suggests that the cloud dissipation has been more effective in the Cep-Cas void.

The alignment of the group of TTSs in the Cep-Cas void has a length of $\sim 8^{\circ}$, which is nearly the same as that of LDN 1333. Given the total mass of the group of TTSs $\left(\sim 8.8 M_{\odot}\right)$ and assuming a SFE of $\sim 1 \%$, about $800 M_{\odot}$ of molecular gas, which is comparable to LDN 1333 , is expected to be detected in ${ }^{13} \mathrm{CO}$ as a physically associated parent cloud of the TTSs. If this is the case, more than $80 \%$ of the molecular mass has been dissipated within a few Myrs.

Grenier et al. (1989) discussed the CO void and concluded that it is likely to be created by a type I supernova $4 \times 10^{4}$ years ago. It seems to be filled with hot plasma seen as a $B$-band soft X-ray excess, although it is not so prominent in the RASS $0.25 \mathrm{keV}$ band. They also claimed that it is rimmed with a radio loop at $408 \mathrm{MHz}$. The estimated distance to a supernova bubble is generally uncertain. After the publication of Grenier et al. (1989), distance measurements to several clouds around the CO void have been carried out (e.g., Kun 1998) and revealed that they are not at the same distance, but either at $200 \mathrm{pc}$ or $300 \mathrm{pc}$. Among the clouds around the CO void, LDN 1241 (marked B in Fig. 2) and LDN 1251 (marked F in Fig. 2) show indication of the interactions with the shock created by the supernova, namely LDN 1241 has a sharp edge on the cloud boundary facing the CO void, while LDN 1251 has elongated cometary shape pointing toward the centre of the $\mathrm{CO}$ void (Sato et al. 1994). Unlike LDN 1241, star formation is active in the dense and massive head of the LDN 1251, suggesting triggered star formation. The distances to both dark clouds are well established to be $\sim 300 \mathrm{pc}$, which supports the distance of $300 \mathrm{pc}$ to the supernova. If the group of TTSs are placed at $300 \mathrm{pc}$ rather than $200 \mathrm{pc}$, they would have been affected by the supernova shock, which caused the rapid dissipation of the parent cloud and the faint clouds in the $\mathrm{CO}$ void have remained as the remnants. Although this scenario requires the revision of the age estimation for the TTSs, the expected time of the supernova explosion is still much later than the formation of the TTSs.

Another possibility is that the TTSs in the CO void were affected by another unknown supernova shock at $200 \mathrm{pc}$ within the last several $\times 10^{5}$ years. Toward the Cep-Cas region, a large arc-like structure is seen in radio continuum called Loop III, whose centre is $(l, b)=\left(124^{\circ},+15^{\circ} .5\right)$ and the estimated distance is $\sim 150 \mathrm{pc}$ (Berkhuijsen et al. 1971; Spoelstra 1972). It is thought to have been created by a supernova explosion. Taking into account its diameter of $\sim 200 \mathrm{pc}$, the group of TTSs and the nearby dark clouds should lie inside the supernova shell. Even though no clear evidence of the interaction can be seen in the nearby clouds, the supernova shock of Loop III is likely to have affected the parent cloud of the TTSs if the distances are nearly the same. LDN $1221\left(l=110^{\circ} .7, b=9.6\right.$, mark L in Fig. 2) also has a head-tail structure (Umemoto et al. 1991), however, it does not point toward the centre of the $\mathrm{CO}$ void or Loop III, and its distance has not been well established yet. In addition to this, several infrared (IR) loops have been identified based on the overlapping $100 \mu \mathrm{m}$ IRAS map in this region (Tóth et al. 1996; Kiss et al. 2004), while the group of TTSs are outside of them. These suggest that there are yet unknown old SNR in the Cep-Cas region.

Alternatively, the TTSs may not be in a group but at different distances. It is possible that some of them are at $300 \mathrm{pc}$ and under the influence of the supernova, and the others are in the foreground. If the faint $\mathrm{CO}$ clouds are truly associated only with the 4 closest stars, the expected original cloud mass 
is about half of the value assumed above. The molecular gas of $\sim 200 M_{\odot}$ could have been dissipated by energetic molecular outflow from the young stars as discussed by Mizuno et al. (1998). Further observational investigations, particularly to obtain accurate distance measurements, will make the formation scenario of the isolated TTSs more clear.

\section{Conclusions}

New T Tauri stars have been searched for in the CepheusCassiopeia star-forming region. Spectroscopic observations toward the ROSAT X-ray sources resulted in detection of $16 \mathrm{Li}$ rich stars. With sufficiently large equivalent widths of $\mathrm{Li}$ $(W(\mathrm{Li}) \gtrsim 0.3 \AA$ ) and relatively bright X-ray luminosities, they are candidate PMS stars. The follow-up photometric observations in $V$ and $I_{\mathrm{C}}$ bands confirmed them as young low-mass stars, namely $\mathrm{T}$ Tauri stars, although 5 of them with relatively weak Li are possibly zero-age main sequence stars or post-TTS and in need of higher resolution spectral observations for their confirmation. The derived spectral types of the new TTSs are from G8 to $\mathrm{K} 7$ and three of them are with yet unconfirmed companion candidate M-type stars. The age and mass of the TTSs are estimated from their location in the HR diagram. Under the assumption of a distance of $200 \mathrm{pc}$, they are all lowmass stars with $0.4 M_{\odot}<M<2 M_{\odot}$ and the majority of them are younger than 10 Myrs.

The distribution of the TTSs is not uniform, but rather grouped. Particularly in the CO void found by Grenier et al. (1989), a group of TTSs are located separated from the ${ }^{13} \mathrm{CO}$ cloud by $\gtrsim 10 \mathrm{pc}$ despite their youth. Compared with other nearby SFRs such as Chamaeleon, the cloud-to-TTS separations are significantly larger for the isolated TTSs. Because of their grouping, an in situ formation model is preferable for them. The total mass of TTSs suggests they were formed in a $\sim 800 M_{\odot}$ molecular cloud, whereas only $\sim 200 M_{\odot}$ of molecular gas now remains in the vicinity of the TTSs. An external disturbance to dissipate the molecular cloud within several $\times 10^{5} \mathrm{yr}$ is required. According to the distance to the TTSs, two scenarios for the history of star formation are suggested: (1) The TTSs in the CO void were formed at $300 \mathrm{pc}$ and were affected by the supernova shock discussed by Grenier et al. (1989); or (2) they are at 200 pc and an unknown supernova explosion such as the progenitor of Loop III are responsible for the parent cloud dissipation.

Acknowledgements. K.T. appreciates the hospitality of the staff of the Calar Alto observatory, Almería, Spain, and the Konkoly observatory, Budapest, Hungary. This publication makes use of database of SIMBAD by CDS, Strasbourg, France. K.T. is financially supported by Japanese Society for the Promotion of Science (JSPS). This work was partly supported by the Hungarian grant OTKA T34584.

\section{References}

Alcalá, J. M., Krautter, J., Schmitt, J. H. M. M., et al. 1995, A\&AS, 114,109

Alcalá, J. M., Krautter, J., Covino, E., et al. 1997, A\&A, 319, 184

Berkhuijsen, E. M., Haslam, C. G. T., \& Salter, C. J. 1971, A\&A, 14, 252
Cohen, J. G., Persson, S. E., Elias, J. H., \& Frogel, J. A. 1981, ApJ, 249, 481

Dame, T. M., Hartmann, D., \& Thaddeus, P. 2001, ApJ, 547, 792

D’Antona, F., \& Mazzitelli, I. 1994, ApJS, 90, 467

Feigelson, E. 1996, ApJ, 468, 306

Feigelson, E. D., \& Decampli, W. M. 1981, ApJ, 243, L89

Grenier, I. A., Lebrun, F., Arnaud, M., Dame, T. M., \& Thaddeus, P. 1989, ApJ, 347, 231

Kenyon, S. J., \& Hartmann, L. 1995, ApJS, 101, 117

Kirkpatrick, J. D., Henry, T. J., \& McCarthy, D. W. 1991, ApJS, 77, 417

Kiss, Cs., Tóth, L. V., Moór, A., et al. 2000, A\&A, 363, 755

Kiss, Cs., Moór, A., \& Tóth, L. V. 2004, A\&A, 418, 131

Kun, M. 1998, ApJS, 115, 59

Kun, M., \& Prusti, T. 1993, A\&A, 272, 235

Kun, M., Obayashi, A., Sato, F., et al. 1994, A\&A, 292, 249

Kun, M., Vinkó, J., \& Szabados, L. 2000, MNRAS, 319, 777

Mamajek, E. E., Lawson, W. A., \& Feigelson, E. D. 1999, ApJ, 516, L77

Martín, E. L., \& Kun, M. 1996, A\&AS, 116, 467

Mizuno, A., Hayakawa, T., Yamaguchi, N., et al. 1998, ApJ, 507, L83

Mizuno, A., Yamaguchi, R., Tachihara, K., et al. 2001, PASJ, 53, 1071

Neuhäuser, R. 1997, Science, 276, 1363

Neuhäuser, R., Sterzik, M. F., Schmitt, J. H. M. M., Wichmann, R., \& Krautter, J. 1995, A\&A, 297, 391

Neuhäuser, R., Torres, G., Sterzik, M. F., \& Randich, S. 1997, A\&A, 325,647

Nikolić, S., Kiss, C., Johansson, L. E. B., Wouterloot, J. G. A., \& Tóth, L. V. 2001, A\&A, 367, 694

Obayashi, A., Fukui, Y., Kun, M., Sato, F., \& Yonekura, Y. 1998, AJ, 115,274

Petrov, G., Seggewiss, W., Dieball, A., \& Kovachev, B. 2001, A\&A, 376, 745

Preibisch, T., Guenther, E., \& Zinnecker, H. 2001, ApJ, 121, 1040

Sato, F., \& Fukui, Y. 1989, ApJ, 343, 773

Sato, F., Mizuno, A., Nagahama, T., et al. 1994, ApJ, 435, 279

Schmitt, J. H. M. M., Fleming, T. A., \& Giampapa, M. S. 1995, ApJ, 450,392

Soderblom, D. R., Jones, B. F., Balachandran, S., et al. 1993, AJ, 106, 1059

Spoelstra, T. A. Th. 1972, A\&A, 21, 61

Sterzik, M., Alcalá, J., Neuhäuser, R., \& Schmitt, J. H. M. M. 1995, A\&A, 297, 418

Tachihara, K., Toyoda, S., Onishi, T., et al. 2001, PASJ, 53, 1081

Tachihara, K., Onishi, T., Mizuno, A., \& Fukui, Y. 2002, A\&A, 385, 909

Torres, G., Neuhäuser, R., \& Guenther, E. W. 2002, AJ, 123, 1701

Tóth, L. V., Kiss, C., \& Moór, A. 1996, The Role of Dust in the Formation of Stars, Proc. of the ESO Workshop Held at Garching, Germany, 11-14 September 1995, ed. H. U. Käufl, \& R. Siebenmorgen (Berlin, Heidelberg, New York: SpringerVerlag), Also ESO Astrophysics Symposia (European Southern Observatory), 125

Umemoto, T., Hirano, N., Kameya, O., et al. 1991, ApJ, 377, 510

Voges, W., Aschenbach, B., Boller, Th., et al. 1999, A\&A, 349, 389

Voges, W., Aschenbach, B., Boller, Th., et al. 2000, Max-Planck-Institut für extraterrestrische Physik, Garching, http://www . xray.mpe.mpg.de/rosat/survey/rass-fsc/

Wichmann, R., Krautter, J., Covino, E., et al. 1997, A\&A, 320, 185

Yonekura, Y., Dobashi, K., Mizuno, A., Ogawa, H., \& Fukui, Y. 1997, ApJS, 110, 21

Zboril, M., Byrne, P. B., \& Rolleston, W. R. J. R. 1997, MNRAS, 284, 685 